\title{
Quantification of Patellar Tendon Reflex by Motion Analysis
}

\author{
L.K. Tham1, N.A. Abu Osman1, K.S. Lim2, B. Pingguan-Murphy1, and W.A.B. Wan Abas1 \\ ${ }_{1}$ Department of Biomedical Engineering, Faculty of Engineering, University of Malaya, Kuala Lumpur, Malaysia \\ 2 Division of Neurology, Faculty of Medicine, University of Malays, Kuala Lumpur, Malaysia
}

\section{INTRODUCTION}

The deep tendon reflexes (DTRs) are automatic responses

of muscle spindles if the tendons were stretched

suddenly [1]. Such reflex can be obtained at different parts

of the body such as the biceps, triceps, knee or ankle. DTRs

are often showed as immediate muscle contraction once tap

is applied on the tendon [1].

Reflexes assessment is the basic test performed in a neurologic

examination, which is an important diagnosis in

identifying diseases or lesions in the nervous system [2].

This is because abnormal reflex responses are generally the signs of diseases in the nervous or muscular system [3].

During a particular assessment, reflex of the responding muscle is observed by the examiner and subsequently graded the response base on the examiner's experience [4].

This in turn leads to variation when a patient is examined by different examiners. Different grading scales are used to grade reflex response at the moment [5], leading to high probability where judgments are conveyed wrongly among medical practitioners.

This study investigated the ability of motion analysis in obtaining quantitative measurements for one of the DTR, the patellar tendon reflex which is commonly known as the knee jerk. Motion analysis is widely applied in area such as sports, rehabilitation, gait analysis, prosthetics and orthotics [6], [7]. Cameras capturing the motion of reflective markers attached on human joints produce 3D motion, allowing detail analysis of the motion at a specific joint. This method 
is hoped to solve problem in reflex assessment by providing objective results for the judgments.

\section{Methods}

\section{A. Subjects}

In this study, 50 subjects consisted of 28 males and 22 females were involved as the subject of patellar tendon reflex tests. The subjects were having age within 20 to 25 years old. Male subjects were having an average height of and weight of $1.72 \pm 0.07 \mathrm{~m}$ and $69.75 \pm 12.18 \mathrm{~kg}$. The mean height and weight of female subjects were $1.57 \pm 0.06$ $\mathrm{m}$ and $52.45 \pm 9.29 \mathrm{~kg}$ respectively. In general, all subjects were categorized under normal range of the Body Mass Index (BMI). All subjects are normal and healthy without any history of neurologic disease in order to avoid possibility in obtaining abnormal reflex response.

\section{B. Patellar Tendon Reflex Test}

The patellar tendon reflex tests were carried out in the Motion Analysis Laboratory under the Department of Biomedical Engineering, University of Malaya, Kuala Lumpur, Malaysia. All tests were recorded and processed using the motion analysis system named Vicon Nexus 1.4.

Prior to any test, body measurements such as height and weight were taken from the subject. Then, setup of the subject was done by attaching sixteen reflective markers on the lower limb following the position stated on the Plug-in-Gait marker placement [8]. The subject was seated on a stool with both leg relaxing and not touching the ground. The positions of patellar tendon of both legs were identified and marks were made on the patellar tendons.

Patellar tendon reflexes were examined by tapping the tendon with a Queen Square reflex hammer. To study the effect of different tapping force on patellar tendon reflex, the 
Queen Square reflex hammer was released at three different angles. Firstly, tendon was tapped with reflex hammer released at $30^{\circ}$ which was named Force 1 . Taps were also applied at $60^{\circ}$ named Force 2 and $90^{\circ}$ which named Force 3.

The hammer was first placed perpendicular to the left patellar tendon. Then, reflex hammer was raised to an angle represented as Force 1 measured using a goniometer. The hammer was released to tap on the patellar tendon. Tapping at a particular angle was repeated for five times. The test was then done with taps of Force 2 and Force 3. The same steps were then repeated for the right patellar tendon. The Jendrassik maneuver was tested by tapping the tendons with the reflex hammer released at $60^{\circ}$. Subject was asked to clench the fingers and concentrating in pulling the fingers [4] during the test.

\section{Analysis}

The motion of knee was analyzed by obtaining the knee joint angles from the tests. Knee angles were compared statistically between genders and different sides of leg. Independent $t$ tests with statistical significance of $P<0.05$ were carried out for these comparisons. The study of different tapping for on reflex responses was done by analyzing the data with one way analysis of variance (ANOVA) and Tukey's HSD $(P<0.05)$ as the post hoc analysis.

\section{RESULTS AND DISCUSSION}

Analysis of the patellar tendon reflex response between males and females as represented in Fig. 1 did not show a constant trend. Female subjects showed significant higher knee angle in tapping with Force 1. On the other hand, male subjects showed significant greater knee angle compared to females at Force 2. Results obtained for Force 2 and Force 3 agreed with an early study which found that males exhibit greater reflex responses at higher tapping strength [9]. 
However, comparison between males and females at Force 3 was not significant statistically due to small sample size involving in the study.

The test on Jendrassik maneuver showed non-significant comparison between genders with tap applied with Force 2 . This was again due to small number of subjects.

Analysis of reflex responses between different sides of body was done as shown in Fig. 2. Non-significant results were found for all pairs of comparison at all tapping angles and the Jendrassik maneuver. Such observations were the actual clinical facts where reflexes must be symmetry in physiologically normal human [4]. Normal subjects involving in the study must show no significant difference in reflex response between the left and the right knee. Asymmetry will be a sign of abnormality and neurologic disorder [10].

Full text is available at :

http://download.springer.com/static/pdf/875/chp\%253A10.1007\%252F978-3-642-217296 52.pdf?auth66=1393724480 718bef5a1f77779a23d9904c975770ba\&ext=.pdf

http://link.springer.com/chapter/10.1007/978-3-642-21729-6 52 